\title{
BMJ Open Can physical testing be used to distinguish between migraine and cervicogenic headache sufferers? A protocol for a systematic review
}

\author{
Ernesto Anarte, ${ }^{1}$ Gabriela Ferreira Carvalho, ${ }^{2}$ Annika Schwarz, ${ }^{3}$ Kerstin Luedtke, ${ }^{2}$ \\ Deborah Falla (i) ${ }^{1}$
}

To cite: Anarte E, Ferreira Carvalho G, Schwarz A, et al. Can physical testing be used to distinguish between migraine and cervicogenic headache sufferers? A protocol for a systematic review. BMJ Open 2019;9:e031587. doi:10.1136/ bmjopen-2019-031587

- Prepublication history and additional material for this paper are available online. To view these files, please visit the journal online (http://dx.doi. org/10.1136/bmjopen-2019031587).

Received 10 May 2019 Revised 09 0ctober 2019 Accepted 16 0ctober 2019

Check for updates

(C) Author(s) (or their employer(s)) 2019. Re-use permitted under CC BY-NC. No commercial re-use. See rights and permissions. Published by BMJ.

${ }^{1}$ Centre of Precision Rehabilitation for Spinal Pain (CPR Spine), School of Sport, Exercise and Rehabilitation Sciences, University of Birmingham, Birmingham, UK ${ }^{2}$ Pain and Exercise Research, Universitat zu Lubeck Sektion Medizin, Lubeck, Germany

${ }^{3}$ Department of Systems Neuroscience, University Hospital Hamburg-Eppendorf, Hamburg, Germany

Correspondence to

Deborah Falla;

d.falla@bham.ac.uk

\section{ABSTRACT}

Introduction Differential diagnosis of migraine and cervicogenic headache (CGH) can be challenging given the large overlap of symptoms, commonly leading to misdiagnosis and ineffective treatment. In order to strengthen the differential diagnosis of headache, previous studies have evaluated the utility of physical tests to examine for musculoskeletal impairment, mainly in the cervical spine, which could be provoking or triggering headache. However, no systematic review has attempted to evaluate whether physical tests can differentiate CGH from migraine or both conditions from asymptomatic subjects.

Methods/analysis A systematic review protocol has been designed and is reported in line with Preferred Reporting Items for Systematic Reviews and Meta-Analyses Protocols (PRISMA-P). A sensitive topic-based search strategy is planned which will include databases, hand searching of key journals and consultation of relevant leading authors in this field. Terms and keywords will be selected after discussion and agreement. Two independent reviewers will perform the search and select studies according to inclusion and exclusion criteria, including any cohort or observational studies evaluating the topic of this review; a third reviewer will confirm accuracy. A narrative synthesis will be developed for all included studies and, if possible, a meta-analysis will be conducted. The overall quality of the evidence will be assessed using the Quality Assessment of Diagnostic Accuracy Studies (QUADAS-2) checklist for diagnostic accuracy studies and the Downs and Black scale for those studies where the QUADAS-2 checklist cannot be applied. Ethics and dissemination Ethical approval is not required since no patient information will be collected. The results will provide a deeper understanding about the possibility of using physical tests to differentiate cervicogenic headache from migraine and from asymptomatic subjects, which has direct relevance for clinicians managing people with headache. The results will be published in a peer-reviewed journal and presented at scientific conferences.

PROSPERO registration number CRD42019135269.

\section{INTRODUCTION}

Headache is one of the most common disorders throughout the world, with $50 \%$ of the
Strengths and limitations of this study

- This is the first systematic review to evaluate whether physical tests can be used to differentiate cervicogenic headache from migraine and both conditions from asymptomatic subjects.

- The results will provide a clearer understanding of the role of the physical assessment of patients with cervicogenic headache and migraineurs, including which tests are more useful to differentiate between the two headache types.

- Synthesis of results will follow recommendations from the COnsensus-based Standards for the selection of health Measurement INstruments (COSMIN) guideline for systematic reviews and the quality of the evidence will be assessed using the Quality Assessment of Diagnostic Accuracy Studies and Downs and Black checklists.

- An initial scoping search suggests that a metaanalysis may not be possible due to heterogeneity of available data.

European population classified as headache sufferers. Not surprisingly, headache is associated with high levels of disability, ${ }^{12}$ poor quality of life and reduced labour productivity. ${ }^{3}$

According to the International Headache Society, headaches can be broadly classified as either primary or secondary. Primary headaches, such as migraine, are headaches not caused by underlying disease or structural problems, whereas secondary headaches are considered symptoms of an underlying and known disorder. ${ }^{4}$ Cervicogenic headache $(\mathrm{CGH})$ is an example of a secondary headache, and in this case the main disorder is a dysfunction within the cervical structures, especially the upper cervical segments. ${ }^{5}$ Convergence of cervical and trigeminal afferents in the trigeminocervical nucleus can potentially explain the referred pain perceived as headache in patients with CGH. ${ }^{5}$ However, the 
convergence of cervical and trigeminal afferents in the trigeminocervical nucleus can be bidirectional, and could also explain why some migraine or tension type headache sufferers perceive neck pain in the absence of cervical musculoskeletal dysfunction. ${ }^{6-8}$ In addition, this interaction in the trigeminocervical complex could trigger additional pain to the head in migraineurs, when central sensitisation develops in the nociceptive trigeminocervical system. ${ }^{9-11}$

The overlap of signs and symptoms between migraine and CGH (online supplementary file 1) contribute to the difficulty in the differential diagnosis of headache types, ${ }^{12} 13$ which become more complex in case of mixed headache presentations. ${ }^{13}$ Indeed, it has been argued that in $\sim 50 \%$ of cases, the diagnosis may be incorrect, ${ }^{14}$ leading to inappropriate treatment choices.

The diagnostic criteria applied to headache both in research and clinical practice, typically adhere to those described by the International Classification of Headache Disorders (International Headache Society, IHS) ${ }^{4}$ and the criteria proposed by the Cervicogenic Headache International Study Group ${ }^{15}$ (CHISG), later re-evaluated by Antonaci $e t a l .^{16}$ In order to strengthen the differential diagnosis of headache, some studies have evaluated the utility of physical tests to examine for musculoskeletal impairments, mainly in the cervical spine, which could be provoking or triggering the headache (eg, manual examination, ${ }^{1718}$ mobility tests ${ }^{19-21}$ or strength evaluation, ${ }^{22} 23$ among others).

Two systematic reviews ${ }^{24}{ }^{25}$ have attempted to assess the usefulness of physical examination and manual examination, respectively, for the differential diagnosis of CGH, and a further systematic review ${ }^{26}$ was directed at elucidating which examination tests can be useful to detect musculoskeletal impairment in migraine sufferers. In addition, another systematic review ${ }^{27}$ assessed diagnostic accuracy of headache measurement instruments. Although for migraine all of these tools were questionnaires, and the diagnostic accuracy of only one physical test was evaluated for CGH. No systematic review has attempted to evaluate whether physical tests can differentiate CGH from migraine and both conditions from asymptomatic individuals. Thus, the purpose of this systematic review will be to determine whether: (1) physical examination, including manual examination, can be used to differentiate people with CGH from asymptomatic individuals, (2) physical examination, including manual examination, can be used to differentiate people with migraine from asymptomatic individuals and (3) physical examination, including manual examination, can be used to differentiate people with CGH from those with migraine. To achieve these purposes, we will collect data about physical examination procedures assessing musculoskeletal dysfunction in migraine sufferers, people with CGH and asymptomatic subjects. The results of this systematic review will have direct relevance for clinicians managing people with headache.

\section{METHODS}

The protocol for this systematic review has been designed following scoping searches of the available literature and is reported in accordance with criteria established by Preferred Reporting Items for Systematic Review and Meta-Analysis Protocols (PRISMA-P) guidelines (online supplementary file 2). ${ }^{28} 29$

\section{Eligibility criteria \\ Inclusion criteria \\ Population}

Any study including the physical examination of an adult population (>18 years old) suffering from $\mathrm{CGH}$, migraine or mixed headaches with possible cervical involvement, as defined by the IHS ${ }^{4}$ and CHISG. ${ }^{15}$ Studies assessing people with a diagnosed cervical pathology will be included in this review. Studies, which include other headache types such as tension-type headache, will be included if data for CGH or migraine are reported independently. For the studies assessing diagnostic accuracy in CGH, we will accept any diagnosis based on the IHS and CHISG with the exception of diagnostic anaesthesic blocks. This diagnosis was defined as acceptable if the headache was intermittent, side dominant, headache of moderate intensity, without side shift and it was required to be preceded by ipsilateral neck pain and precipitated or aggravated by neck movement or posture, fulfilling five out of seven criteria outlined by Antonaci, ${ }^{16}$ as proposed in other studies. In relation to diagnostic accuracy studies for migraine, diagnosis must be based on the IHS criteria ${ }^{11}$ for migraine without aura. In addition, this diagnosis will be considered as acceptable if it does not meet the IHS criteria for other forms of headache. Finally, asymptomatic subjects will be defined as those people who had no history of subjective features of CGH, migraine, migraine with aura headache, episodic headache and neck pain or stiffness.

\section{Intervention}

The intervention of interest is the physical examination directed at evaluating the presence or absence of cervical musculoskeletal impairment in CGH and/or migraine sufferers. This includes studies examining cervical articular, muscular and/or neural structures.

As described previously, ${ }^{24}$ physical examination tests are defined as clinician performed tests or measures that were designed to obtain a diagnosis or detect an impairment.

\section{Comparison}

The studies included must compare physical examination findings between (a) CGH and asymptomatic controls; (b) CGH and migraine; (c) migraine and asymptomatic controls and/or (d) CGH, migraine and asymptomatic controls.

\section{Outcome measures}

Any physical examination or test designed to evaluate the musculoskeletal system including, but not limited to, range of motion, muscular strength and endurance, 
reproduction or resolution of symptoms by manual examination, tenderness palpation, proprioceptive measures.

\section{Study design}

Case-control studies will be the study design of reference for this review. Any cohort study or observational study design will also be included. If diagnostic tests were performed prior to an intervention, randomised controlled trials will be also included. Case studies and previous literature reviews including systematic reviews and meta-analyses will be excluded.

\section{Measurement properties}

Any observational study evaluating one or more measurement property will be included. If possible, data on diagnostic accuracy will be collected in this review. For diagnostic accuracy, we will collect sensitivity (SN), specificity (SP), positive and negative likelihood ratios (LR+ and LR-) and positive and negative predictive values (PPV and NPV). SN is defined as the percentage of people who test positive for a specific diagnosis among a group of people who have the diagnosis. SP is the percentage of people who test negative for a specific diagnosis among a group of people who do not have the diagnosis. A LR+ identifies the strength of a test in determining the presence of a finding. A higher LR+ reflects a stronger ability to detect the condition when the test is positive. The LRidentifies how much the odds of the diagnosis decrease when a test is negative. The lower the value, the better the ability of the test to determine the chance, the diagnosis is actually present in the event the finding is negative. ${ }^{30}$ Finally, PPV and NPV are the proportions of individuals with positive and negative test results, respectively, who are correctly diagnosed. ${ }^{31}$

\section{Timing and setting}

No restriction on setting or timing will be imposed.

\section{Exclusion criteria}

People suffering of a serious disease or another diagnosed headache condition not described in the inclusion criteria will be excluded. Individuals with a history of head or neck trauma will also be excluded. In addition, all studies which are not written in English will be excluded.

\section{Source of information}

The search will include articles from the following databases: Medline, PubMed, Web of Science, Scopus, EMBASE and CINAHL. Search strategies in these databases will include medical subject headings (MESH) and natural language terms. In addition, we will perform hand searching of specific journals where we found potentially eligible studies in an initial scoping search: Cephalalgia, Headache, The Journal of Headache and Pain, Current Pain and Headache Reports, Manual Therapy, Musculoskeletal Science and Practice, Physical Therapy and Journal of Manipulative and Physiological Therapeutics. This systematic review will be conducted between October 2019 and January 2020.

\section{Search strategy}

This search will be completed by two reviewers (EA and GFC). An initial MEDLINE search strategy will be defined, including MESH keywords and natural language term combinations that will be adapted to other databases. The specific search strategies will be developed in consensus by all authors and facilitated by a health science librarian. Search strategy will be developed between 30 September 2019 and 31 October 2019.

Example of search strategy used in Medline

( ((cervicogenic headache [Mesh Term]) OR migraine

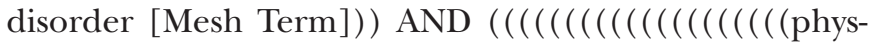
ical diagnosis [Mesh Term]) OR physical examination [Mesh Term]) OR manual examination [All Fields]) OR physical tests [All Fields]) OR cervical musculoskeletal impairments [All Fields]) OR endurance[All Fields]) OR cranio-cervical flexion [All Fields]) OR muscle function [All Fields]) OR flexion-rotation [All Fields]) OR joint position error [All Fields]) OR joint position sense [All Fields] OR tenderness [All Fields]) OR trigger point [All Fields]) OR joint [All Fields]) OR mobility [All Fields]) OR range of motion [All Fields]) OR pressure pain threshold [All Fields]) OR posture [All Fields]) OR muscle strength [All Fields]))

Results of the search and the process of screening and selecting studies for inclusion in the review will be presented in a flow diagram described by PRISMA-P guidelines. $^{2728}$

\section{Data management}

All results will be uploaded and stored on Mendeley, a desktop and web programme. Any duplicates will be identified and removed. Each reviewer will save the results in an individual folder.

\section{Study selection}

Two reviewers (EA and GFC) will independently screen titles/abstracts against the prespecified inclusion/exclusion criteria and subcategorise into include/exclude/ unsure. For those that meet the inclusion criteria, the full texts will be obtained. Moreover, if any uncertainty exists, then the full text will be retrieved for further clarification. If needed, the authors of the original work will be contacted. Screening of full texts will be conducted in the same manner using the predefined criteria and conducted independently.

Articles will be included when eligibility is confirmed by both reviewers. Any disagreement between the two reviewers will be first discussed in a consensus meeting between the reviewers, and if no agreement can be made, an independent reviewer (KL) will be sought to decide about inclusion. Information on excluded studies and reasons for exclusion will be collated and reported. Reviewers will not be blinded to journal titles and study authors.

\section{Data collection and data items}

A standardised form will be used to extract information from the included studies. Both reviewers will 
independently extract information, with one reviewer subsequently collating the information. Any disagreements between the reviewers will be discussed and/or reviewed by a third reviewer.

Data extracted will include the following summary data: title and authors, year of publication, sample characteristics, study design, diagnostic criteria for each group, reported time point in migraine cycle, total number of intervention groups, specific physical examination tests, assessment protocol, outcomes of interest, measurement properties assessing statistical methods used and results.

\section{Outcomes and priorisation}

Although resolution of headache after neck treatment has been accepted as a confirmation for CGH diagnosis; ${ }^{4}$ a gold standard physical test which could be used at the initial assessment for the differential diagnosis of CGH and migraine has not been identified so far. Therefore, we aim to identify a measure of physical impairment in the neck that will allow clinicians to differentially diagnosis between these two headache types, although no prioritisation can be defined.

\section{Risk of bias}

As done in a recent systematic review ${ }^{26}$ and recommended by the Cochrane Handbook for Systematic Reviews of Interventions, ${ }^{32}$ risk of bias will be assessed (except for the diagnostic accuracy studies) using the Downs and Black Scale, ${ }^{33}$ but excluding those items that have been proposed to assess interventions. Each trial will be independently assessed and scored by two reviewers (AS and $\mathrm{KL}$ ) in terms of risk of bias, and a third reviewer (DF) will intervene in the case of disagreement. A quality index will be calculated, indicating low, moderate or high risk of bias. The Cohen kappa coefficient will be calculated to express the agreement between reviewers. The same procedure, by the same reviewers, will be used to assess risk of bias of diagnostic accuracy studies. However, in this case, the Quality Assessment of Diagnostic Accuracy Studies (QUADAS-2) ${ }^{34}$ tool will be used, which was developed to evaluate quality of this type of study, separating risk of bias and concerns regarding applicability. Using this tool, risk of bias will be judged as 'low', 'high' or 'unclear'. ${ }^{34}$

\section{Data synthesis}

If possible, a meta-analysis will be conducted; if not possible due to heterogeneity of populations studied, a narrative synthesis will be conducted for all included studies. This synthesis will include presentation of data in a summary and in tables. Synthesis of results will follow recommendations from the COSMIN guideline for systematic reviews. The quality of the evidence will be assessed using the QUADAS-2 ${ }^{34}$ scale for the quality of diagnostic accuracy studies and the Downs and Black Scale ${ }^{33}$ for the studies where the QUADAS-2 checklist cannot be used. The reviewers will agree on which checklist to use for each study.

\section{Metabiases}

In order to determine whether any reporting bias is present, a detailed search for unpublished studies will be conducted, for example, accessing any past conference proceedings in the last 10 years, and internet searches in clinical trial registries, with consistency between protocols and published studies examined, if protocols are available.

\section{Confidence in cumulative evidence}

As this systematic review assess the confidence of physical tests, two tools will be used to investigate the strength and quality of evidence. The QUADAS-2 scale for diagnostic accuracy studies and in those studies where this checklist cannot be used, Downs and Black Scale will be applied.

\section{Patient and public involvement}

The research question in this study was developed following consultation and discussion with headache patients. Patients will not be involved in the analysis and data collection of the systematic review.

\section{Clinical implications}

Headache is a common disorder with a significant impact on the quality of life of sufferers. ${ }^{1-3}$ Due to the large symptomatic overlap between different headache types, differential diagnosis can be challenging, particularly between CGH and migraine ${ }^{12-14}$ which can lead to an inappropriate choice of treatment.

Physical tests have been developed and described to facilitate the identification of musculoskeletal impairments which could facilitate differential diagnosis between CGH and other forms of headache, in order to confirm the presence of cervical musculoskeletal dysfunction which could be triggering the headache. However, controversy remains about the utility of the physical examination to differentiate migraine from CGH especially since many patients with migraine also suffer from neck pain and have shown cervical musculoskeletal impairment in the past. This systematic review will determine whether physical tests can differentiate CGH from migraine and from asymptomatic subjects which has direct relevance for clinicians managing people with headache.

\section{Twitter Deborah Falla @Deb_Falla}

Contributors EA and DF formulated the focus of the systematic review. EA drafted the initial version of the protocol manuscript with DF. GFC, AS and KL provided guidance on topic, methodology and analyses. All authors reviewed and commented on each draft of the protocol. All authors have approved the final manuscript.

Funding The authors have not declared a specific grant for this research from any funding agency in the public, commercial or not-for-profit sectors.

Competing interests None declared.

Patient consent for publication Not required.

Provenance and peer review Not commissioned; externally peer reviewed.

Open access This is an open access article distributed in accordance with the Creative Commons Attribution Non Commercial (CC BY-NC 4.0) license, which permits others to distribute, remix, adapt, build upon this work non-commercially, and license their derivative works on different terms, provided the original work is 
properly cited, appropriate credit is given, any changes made indicated, and the use is non-commercial. See: http://creativecommons.org/licenses/by-nc/4.0/.

\section{ORCID iD}

Deborah Falla http://orcid.org/0000-0003-1689-6190

\section{REFERENCES}

1 Stovner L, Hagen K, Jensen R, et al. The global burden of headache: a documentation of headache prevalence and disability worldwide. Cephalalgia 2007;27:193-210.

2 Stovner LJ, Andree C. Prevalence of headache in Europe: a review for the Eurolight project. J Headache Pain 2010;11:289-99.

3 Stovner LJ, Hagen K. Prevalence, burden, and cost of headache disorders. Curr Opin Neurol 2006;19:281-5.

4 Headache classification Committee of the International headache Society (IHS) the International classification of headache disorders, 3rd edition. Cephalalgia 2018;38:1-211.

5 Bogduk N, Govind J. Cervicogenic headache: an assessment of the evidence on clinical diagnosis, invasive tests, and treatment. Lancet Neurol 2009;8:959-68.

6 Bartsch T, Goadsby PJ. Increased responses in trigeminocervical nociceptive neurons to cervical input after stimulation of the dura mater. Brain 2003;126:1801-13.

7 Bartsch T. Migraine and the neck: new insights from basic data. Curr Pain Headache Rep 2005;9:191-6.

8 Ashina S, Bendtsen L, Lyngberg AC, et al. Prevalence of neck pain in migraine and tension-type headache: a population study. Cephalalgia 2015;35:211-9.

9 Bartsch T, Goadsby PJ. The trigeminocervical complex and migraine: current concepts and synthesis. Curr Pain Headache Rep 2003;7:371-6.

10 Bartsch T, Goadsby PJ. Anatomy and physiology of pain referral patterns in primary and cervicogenic headache disorders. Headache Currents 2005;2:42-8.

11 Bartsch T, Goadsby PJ. Stimulation of the greater occipital nerve induces increased central excitability of dural afferent input. Brain 2002;125:1496-509.

12 Becker WJ. Cervicogenic headache: evidence that the neck is a pain generator. Headache 2010;50:699-705.

13 Blumenfeld A, Siavoshi S. The challenges of Cervicogenic headache. Curr Pain Headache Rep 2018;22:47.

14 Pfaffenrath V, Kaube $\mathrm{H}$. Diagnostics of cervicogenic headache. Funct Neurol 1990;5:159-64.

15 Sjaastad O, Fredriksen TA, Pfaffenrath V. Cervicogenic headache: diagnostic criteria. The Cervicogenic headache International Study Group. Headache 1998;38:442-5.

16 Antonaci F, Ghirmai S, Bono G, et al. Cervicogenic headache: evaluation of the original diagnostic criteria. Cephalalgia 2001;21:573-83.

17 Zito G, Jull G, Story I. Clinical tests of musculoskeletal dysfunction in the diagnosis of cervicogenic headache. Man Ther 2006;11:118-29.
18 Watson DH, Drummond PD. Head pain referral during examination of the neck in migraine and tension-type headache. Headache 2012;52:1226-35.

19 Hall TM, Briffa K, Hopper D, et al. Comparative analysis and diagnostic accuracy of the cervical flexion-rotation test. $J$ Headache Pain 2010;11:391-7.

20 Ogince M, Hall T, Robinson K, et al. The diagnostic validity of the cervical flexion-rotation test in $\mathrm{C} 1 / 2$-related cervicogenic headache. Man Ther 2007;12:256-62.

21 Luedtke K, Starke W, May A. Musculoskeletal dysfunction in migraine patients. Cephalalgia 2018;38:865-75.

22 Jull G, Amiri M, Bullock-Saxton J, et al. Cervical musculoskeletal impairment in frequent intermittent headache. Part 1: subjects with single headaches. Cephalalgia 2007;27:793-802.

23 Amiri M, Jull G, Bullock-Saxton J, et al. Cervical musculoskeletal impairment in frequent intermittent headache. Part 2: subjects with concurrent headache types. Cephalalgia 2007;27:891-8.

24 Rubio-Ochoa J, Benítez-Martínez J, Lluch E, et al. Physical examination tests for screening and diagnosis of cervicogenic headache: a systematic review. Man Ther 2016;21:35-40.

25 Howard PD, Behrns W, Martino MD, et al. Manual examination in the diagnosis of cervicogenic headache: a systematic literature review. $J$ Man Manip Ther 2015;23:210-8.

26 Szikszay TM, Hoenick S, von Korn K, et al. Which examination tests detect differences in cervical musculoskeletal impairments in people with migraine? A systematic review and meta-analysis. Phys Ther 2019;99:549-69.

27 van der Meer HA, Visscher CM, Vredeveld T, et al. The diagnostic accuracy of headache measurement instruments: a systematic review and meta-analysis focusing on headaches associated with musculoskeletal symptoms. Cephalalgia 2019;39:1313-32.

28 Moher D, Shamseer L, Clarke M, et al. Preferred reporting items for systematic review and meta-analysis protocols (PRISMA-P) 2015 statement. Syst Rev 2015;4:1.

29 Shamseer L, Moher D, Clarke M, et al. Preferred reporting items for systematic review and meta-analysis protocols (PRISMA-P) 2015: elaboration and explanation. BMJ 2015;349:g7647.

30 Cook C, Hegedus E. Diagnostic utility of clinical tests for spinal dysfunction. Man Ther 2011;16:21-5.

31 Cook C, Hegedus E. Orthopaedic physical examination tests: an evidence based approach. Upper Saddle River, NJ: Prentice Hall, 2008. ISBN: 10: 0131791001 ISBN-13: 978-0131791008.

32 Higgins JPT, Green S. Cochrane Handbook for systematic reviews of interventions. version 5.1.0. The Cochrane collaboration, 2011. Available: http://handbook.cochrane.org [Accessed 12 Nov 2018].

33 Downs SH, Black N. The feasibility of creating a checklist for the assessment of the methodological quality both of randomised and non-randomised studies of health care interventions. J Epidemiol Community Health 1998;52:377-84.

34 Whiting PF, Rutjes AWS, Westwood ME, et al. QUADAS-2: a revised tool for the quality assessment of diagnostic accuracy studies. Ann Intern Med 2011;155:529-36. 